\title{
El método R5 de Práctica Reflexiva. Evaluación de su impacto en la formación inicial de maestros
}

\section{The R5 method of Reflective Practice. Evaluation of its impact on initial teacher training}

\author{
Àngels Domingo Roget ${ }^{1}$ \\ 1Plataforma Internacional Práctica Reflexiva (adomingo@practicareflexiva.pro)
}

Recibido el 30 de marzo de 2018; revisado el 25 de abril de 2018; aceptado el 25 de abril de 2018; publicado el 1 de junio de 2018

\section{RESUMEN:}

Partiendo de la investigación doctoral de la autora y su subsiguiente experiencia en el ámbito de la Práctica Reflexiva, se presenta el estudio investigativo enmarcado en el Practicum de estudiantes de Grado de Educación Infantil y Primaria de la Facultad de Educación de la Universitat Internacional de Catalunya (UIC), con el objetivo de destacar la importancia de una práctica reflexiva en la formación de maestros reflexivos para mejorar la gestión del aula y facilitar la resolución de los problemas que se van presentando, por medio de un análisis en el que se integra la teoría con la práctica.

La investigación inició con su correspondiente contextualización y la creación de una propuesta del Método R5 de Práctica Reflexiva, el cual fue aplicado y valorado por los estudiantes del Practicum bajo la supervisión de sus tutores, lo que permitió recoger y analizar distintas categorías de Formación Reflexiva, Articulación de Teoría y Práctica, Método R5 y Uso de Portafolio. Los resultados fueron favorables, mostrando que la Práctica Reflexiva a través del Método R5 y los demás elementos mencionados, contribuyen al desarrollo de la capacidad reflexiva de quienes los aplican mejorando su profesionalización docente.

\section{ABSTRACT:}

Starting from the author's doctoral research carried out with students in the Practicum to get their Early Childhood and Primary Education Degree at the Education School of the International University of Catalonia, as well as the author's subsequent experience in the field of Reflective Practice, the research study has the aim of highlighting the importance of reflective practice and the training of reflective teachers in order to improve the classroom management and facilitate the resolution of problems that arise through an analysis in which theory and practice are integrated.

The research began with its corresponding contextualization and the creation of a proposal of the R5 Method of Reflective Practice, which was applied and valued by students of the Practicum under the supervision of their teacher-tutors, which allowed to collect and analyze data under the headings of Reflective Training, Articulation of Theory and Practice, R5 Method and the Use of a Portfolio. The results were favorable showing that the Reflective Practice through the R5 Method and the other elements mentioned, contribute to the development of the reflexive capacity of those who apply them by improving their teaching professionalization.

KEYWORDS: REFLECTIVE PRACTICE, TRAINING, THEORY, PRACTICE, PORTFOLIO, REFLECTION. 


\section{INTRODUCCIÓN}

La presente experiencia sobre Práctica Reflexiva se circunscribe al ámbito universitario y el marco teórico se nutre de mi anterior investigación doctoral (Domingo, 2013) sobre la efectividad formativa del aprendizaje experiencial en la Educación Superior.

En los últimos 10 años he seguido investigando con modelos innovadores de formación docente basados en la reflexión sobre la práctica en diversos contextos internacionales y con administraciones educativas de distintos países: España (2008-2018), Chile (2017), Argentina (2017 y 2018), Colombia (2015 y 2018), México (2015), Perú (2016), Panamá (2010 a 2015), Andorra (2018), entre otros.

La presente investigación se enmarca en el ámbito curricular del Practicum de los estudiantes de Grado de Educación Infantil y Primaria de la Facultad de Educación de la Universitat Internacional de Catalunya, (UIC), universidad privada ubicada en Barcelona (España).

La finalidad principal de esta experiencia de Práctica reflexiva (PR) es lograr en los estudiantes del Grado de Educación Infantil y Primaria una mayor profundidad en la articulación de su conocimiento teórico y práctico, aspecto intrínsecamente relacionado con su profesionalidad docente.

Algunos detalles más concretos del contexto de esta experiencia son:

a) Se planteó como una innovación de participación voluntaria pero exclusivamente para los estudiantes de la asignatura de Practicum del tercer curso de Educación Primaria e Infantil.

b) La muestra de esta experimentación la conformaron 15 alumnos, seleccionados mediante la técnica de muestreo no probabilístico, o muestra dirigida, que supone un procedimiento de selección informal. En este caso todas eran mujeres y, yo personalmente, fui la docente-investigadora que ha llevado a cabo la experiencia.

En la figura 1 se muestra cómo se estructuró la experiencia de Práctica reflexiva en el marco del Practicum de los futuros maestros.

\section{MATERIAL Y MÉTODO}

Se presenta a continuación el diseño del trabajo de campo indicando las fases del trabajo de aplicación y experimentación del Método R5, adaptado de Domingo (2013).

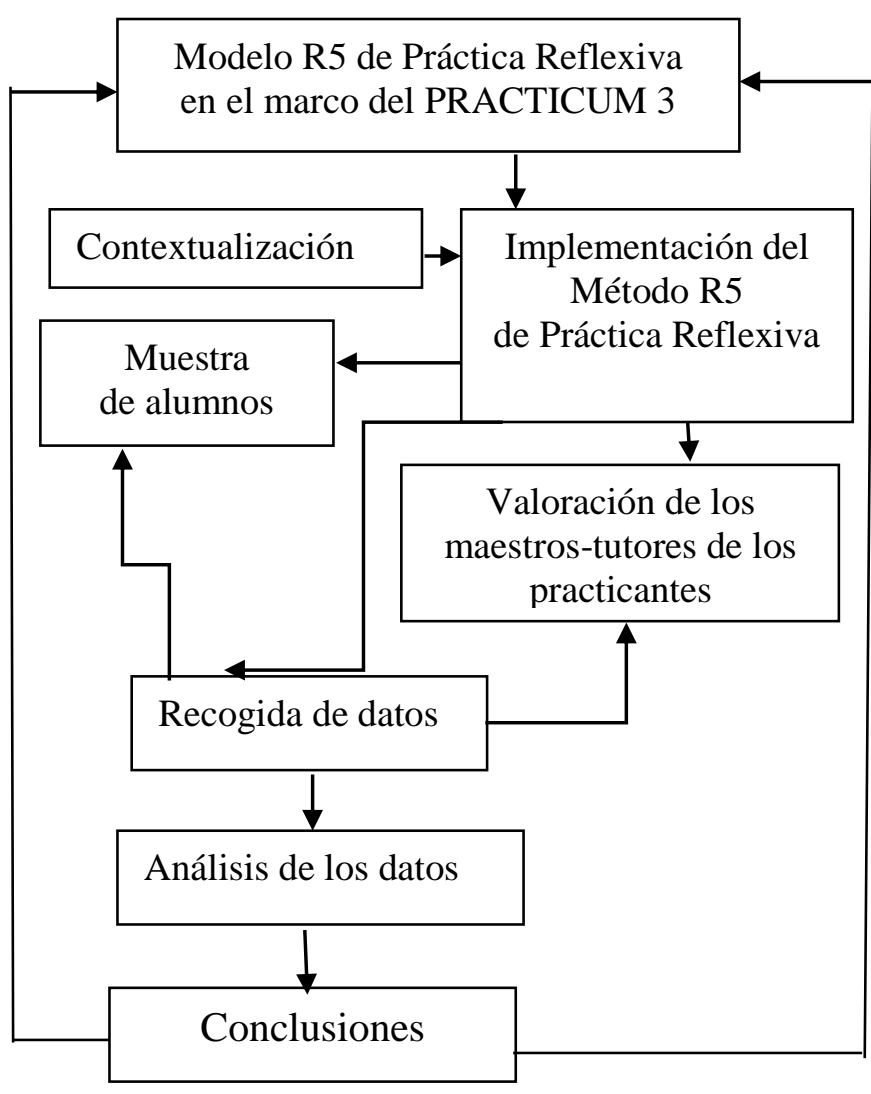

Figura 1: estructura de la investigación

Los objetivos formativos de la experiencia, objeto de esta investigación, en relación con los participantes son los siguientes:

- Formar maestros reflexivos capaces de resolver las situaciones prácticas como profesionales expertos.

- Enseñar a los maestros a sumergirse en la espiral continua de acción-reflexión- acción, que integra bidireccionalmente la teoría y la práctica, el conocimiento formal y el conocimiento práctico, el criterio científico y el compromiso ético y social.

- Proporcionar al maestro una metodología que mejore su capacidad de gestionar adecuadamente en el aula escolar: la contextualización, la complejidad y la toma de decisiones en situaciones de incertidumbre.

- Aumentar en los futuros maestros la preparación metodológica para innovar e investigar a partir de su propia práctica

- Experimentar las ventajas de la reflexión colaborativa. 
Se describen a continuación las fases del trabajo experimental:

Fase 1. Preparación de los materiales: se elaboró un programa formativo para los estudiantes, el cual incluyó documentos, guías, materiales, modelo de $\mathrm{PR}$, cuestionarios.

Fase 2. Estudios de muestreo: se seleccionó la muestra de estudiantes.

Fase 3. Formación de las estudiantes, antes del periodo de prácticas escolares: se realizaron 3 seminarios y entrevistas:

- Seminario grupal $n^{\circ} 1$ : presentación de la prueba piloto a las 15 alumnas voluntarias que componen la muestra

- Seminario grupal $n^{o}$ 2: presentación del Método R5

- Seminario grupal $n^{o}$ 3: presentación y entrega de instrumentos y materiales para el estudiante y su tutor de la escuela. Entrega del guion del portafolio $^{1}$

- Entrevistas: tutorización con las alumnas que lo solicitaron antes de iniciar las prácticas.

Fase 4. Reunión semanal de $2 \mathrm{~h}$. en la universidad durante las 5 semanas del periodo de prácticas: seminarios grupales y tutorización online. Seis Sesiones de Práctica Reflexiva Colaborativa (Domingo, 2017) con modelo R5. Presentación de casos para la reflexión: interacción y nuevas propuestas de intervención educativa.

Fase 5. Aplicación del cuestionario final individual al terminar el periodo de prácticas: cumplimentaron el cuestionario final de forma individual.

Fase 6. Recogida de datos: recogida de la valoración general del modelo de PR de los maestros que tutorizaron a las estudiantes y elaboración final del Portafolio por parte de las participantes para su entrega y evaluación.

Fase 7. Obtención de resultados: los datos se obtuvieron, se analizaron descriptivamente e interpretativamente y se valoró el modelo nuevo de PR.

Se muestra a continuación la organización temática de los seminarios y de los trabajos de las alumnas en relación con la investigación. Cada seminario tuvo una duración de 10 a 12 horas aproximadamente y se llevaron a cabo entre los meses de marzo a junio del 2016:

\footnotetext{
${ }^{1}$ Lo ha de elaborar el estudiante durante sus prácticas y constituye a la vez una estrategia de aprendizaje experiencial y uno de los instrumentos de evaluación del Practicum del tercer curso.
}

Tabla 1. Tareas de los participantes

1er. Seminario informativo: presentación prueba
piloto
Entrevista y documentación en la escuela de
prácticas
Inicio de Prácticas escolares
$2^{\circ}$ Seminario de PR: relación alumno-maestro
3er. Seminario de PR: procesos didácticos
$4^{\circ}$ Seminario de PR: atención a la diversidad
$5^{\circ}$ Seminario de PR: aspectos organizativos
$6^{\circ}$ Seminario Final de PR: otros casos y
valoración
Entrevista individual de cada participante con la
tutora-investigadora y devolución de los
portafolios evaluados

Con la intención de facilitar a los estudiantes una reflexión semanal asequible y acotada, se acordó una temática orientativa de interés:

- Caso práctico - $1^{\mathrm{a}}$ semana: relación o interacción alumno/docente-practicante.

- Caso práctico - $2^{\mathrm{a}}$ semana: procesos de enseñanza aprendizaje.

- Caso práctico - $3^{\mathrm{a}}$ semana: tratamiento de la diversidad.

- Caso práctico - $4^{\mathrm{a}}$ semana: aspectos organizativos.

- Caso práctico- optativo: tema libre

\subsection{Instrumentos}

Se exponen a continuación los instrumentos diseñados para la recolección de resultados con una somera descripción de cada uno de ellos y el ámbito que analiza:

\section{Cuestionario inicial}

Descripción y finalidad: instrumento formal para realizar una evaluación inicial y delinear y conocer las características de la muestra.

Ámbito de análisis: conocimientos previos y expectativas de los estudiantes acerca de la PR.

\section{Modelo para la reflexión individual y grupal: Método R5}

Descripción y finalidad: presentación, justificación y experimentación del Método R5.

Ámbito de análisis: fragmentación explicativa del modelo para aprender de su propia práctica. 


\section{Registro de información}

Descripción y finalidad: desarrollo de los seminarios semanales en los que los participantes presentan situaciones reales vividas en el aula y alas que se aplica el Método R5, narrando su reflexión individual y en busca de una reflexión compartida y la reconceptualización de su aprendizaje experiencial y reflexivo.

Ámbito de análisis: Dinámica de la participación, contraste e interacción entre los practicantes y clima emocional de cada seminario.

\section{Documentos de formación}

Descripción y finalidad: conocimientos teóricos y prácticos aplicados al aula escolar.

Ámbito de análisis: vertebración entre teoría y práctica en los estudiantes de la muestra.

\section{Hoja de evaluación y valoración del maestro- tutor}

Descripción y finalidad: valoración evaluativa del maestro sobre el practicante y el modelo nuevo de PR.

Ámbito de análisis: valoración general del maestro sobre la PR del practicante tutorizado.

\section{Cuestionario final estudiantes}

Descripción y finalidad: instrumento formal de ítems organizados en 4 bloques temáticos y con preguntas.

Ámbito de análisis: percepción y valoración de los alumnos del modelo propuesto de PR.

\section{Método R5 de Práctica Reflexiva}

El proceso reflexivo que sustenta el Método $R^{5}$ (Domingo 2013) sigue la siguiente secuencia:

a) Situación práctica del aula (positiva o negativa).

b) Reconstrucción a posteriori de una situación, análisis e identificación de elementos.

c) Elaboración de conclusiones provisionales sobre:

1.- Posible intervención educativa en la situación.

2.- Revisión del propio conocimiento práctico.

d) Reflexión compartida a través de la interacción, contraste y verbalización.

e) Contraste de la situación con la teoría. Detección de vacíos teóricos.

f) Revisión de las conclusiones provisionales y elaboración de las definitivas.

g) Reelaboración del plan de intervención en el aula. h) Aspectos de mejora personal en relación con las propias teorías implícitas y/o el pensamiento práctico individual.

Estas fases estructuradas de forma cíclica configuran el denominado Método $\mathrm{R}^{5}$, un modelo innovador que guía la reflexión sistemática a través de cinco fases y optimiza el aprendizaje a partir de la experiencia (figura 2).

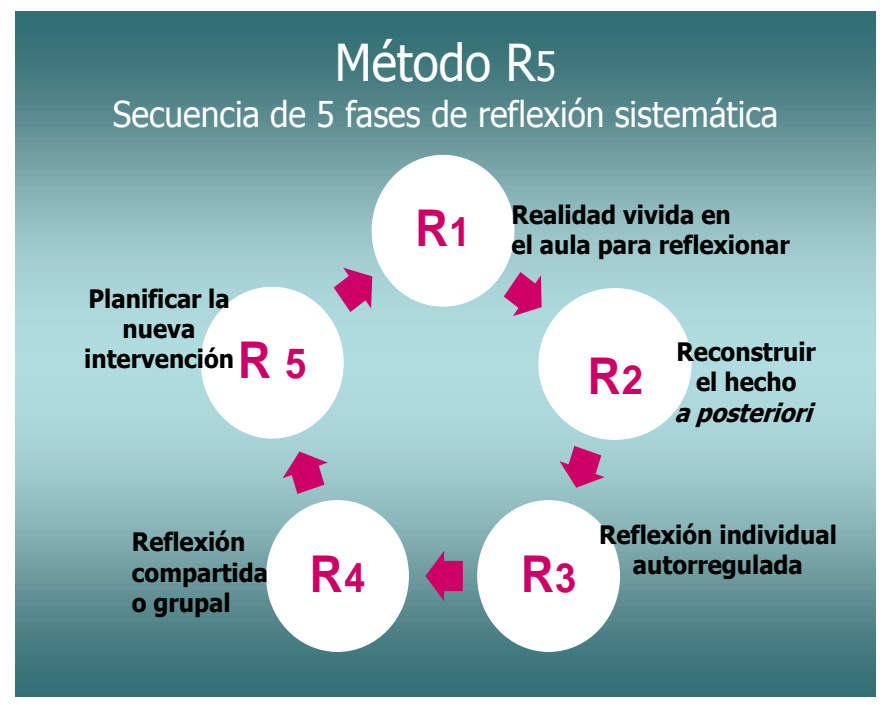

Figura 2. Método R5

El Método $R 5$ recibe este nombre al proponer cinco fases reflexivas. La $\mathrm{R}$ proviene del término "Reflexión" y el 5 procede del $n^{\circ}$ de las fases de la secuencia reflexivo-cíclica configuradoras del método innovador.

La configuración del método R5 presenta una estructura cerrada que pone de manifiesto uno de los aspectos primordiales del modelo: expresa gráficamente que el objetivo de la PR es la mejora de la propia práctica del docente; por tanto, el método parte de la práctica y vuelve a esta para mejorarla. De este modo, la propuesta deviene una metodología que contribuye directamente a la profesionalización del maestro en la resolución de situaciones de carácter práctico.

Se detalla el contenido y características de las fases que presenta la figura 2 .

$\mathbf{R}^{\mathbf{1}}$. Fase de reflexión 1. Seleccionar una situación práctica del aula que se quiera analizar. Puede tratarse de una actuación que ha conducido a un fracaso o a un éxito, un resultado positivo o negativo. La situación debe ser concreta y vivida.

$\mathbf{R}^{2}$. Fase de reflexión 2. Reconstruir el hecho $a$ posteriori. Para ello es necesario recordarla y escribirla. El hecho de escribir centra la mente y depura la acción sobre la cual reflexionar. Al mismo tiempo, es necesario destacar aquello que ha actuado como detonante y que ha hecho salir de la rutina al 
docente, obligándonos a reflexionar en plena acción y a realizar cambios, tomar decisiones y replantear su acción, su intervención y cuestionarse su planificación inicial.

$\mathbf{R}^{3}$. Fase de reflexión 3. Reflexión individual autorregulada.

En esta fase se aconseja la secuencia reflexiva del profesional reflexivo de Schön (1987):

- Conocimiento en la acción. Conocimiento y bagaje formativo personal de cada docente cognitivo, vivencial, teórico, experiencial, emocional, etc.- que se pone en acción de forma espontánea en la acción-profesión y que no explicitamos verbalmente.

- Reflexión en la acción. Cuando un desencadenante (hecho o percepción) provoca que el docente, sin detener la acción, deba cambiar su rutina. El detonante que ha captado le hace reflexionar mientras actúa, revisar, decidir qué hacer y decidir cómo intervenir y solucionar un problema práctico inesperado.

- Reflexión sobre la "reflexión en la acción". Se analiza a posteriori la reflexión sobre la acción y se decide cómo mejorar la intervención.

$R^{4}$. Fase de reflexión 4. Práctica reflexiva colaborativa.

Interacción y contraste con los otros colegas, con el conocimiento teórico colectivo y con uno mismo. En esta fase momento se activa y despliega la denominada Zona de Desarrollo Próximo o Potencial (Vygotski, 1979) fundamentada en la teoría socio-cultural del desarrollo humano de dicho autor.

$\mathbf{R}^{5}$. Fase de reflexión 5. Optimizar la propia práctica.

1. ¿Qué he aprendido de esta situación práctica?

2. ¿Qué deseo modificar y qué deseo mantener en mi siguiente intervención?

3. ¿Con qué conocimientos teóricos relaciono la experiencia vivida en el aula?

4. ¿Cómo influye o modifica esta experiencia mis conocimientos teóricos?

5. ¿Qué vacíos intelectuales he detectado en mi formación?

6. ¿Qué elementos subyacentes -conscientes o inconscientes- considero que debería variar o superar o quizás mantener?

7. Si volviera a repetirse este caso parecido ¿haría algo distinto de lo que hecho como resultado de esta práctica reflexiva realizada?
Al presentar el método ha quedado integrado como parte esencial del mismo la fase R4 en que la reflexión grupal sobre la práctica se ve enriquecida por las distintas perspectivas de los practicantesdocentes que participan en la sesión formativa. Por ello enmarcamos el método R5 en la modalidad de Práctica Reflexiva Colaborativa (Domingo y Anijovich, 2017).

Si es formativa la reflexión de carácter individual cabe señalar que la efectividad formativa de la práctica reflexiva crece de forma exponencial cuando en el modelo contempla la reflexión de y con otros colegas que intercambian miradas y amplían percepciones y conocimientos. En esta nueva situación el proceso de reflexión, a partir de la interacción y contraste con otros colegas, agrega riqueza y sinergia reflexiva en los participantes.

La PR, como se ha expuesto y representado gráficamente, parte y se nutre de las situaciones vivas y problemáticas o exitosas de los escenarios profesionales -en nuestro caso el aula escolar de los practicantes- y busca la resolución de las mismas y lo hace enriqueciendo al propio docente-practicante que, a lo largo del proceso reflexivo individual y colaborativo, aprende, desaprende lo necesario, reconstruye su conocimiento práctico y reformula sus conocimientos teóricos, iluminados ahora por una perfecta simbiosis entre teoría y práctica, ambas al servicio de los problemas diarios del aula. De este modo, en el maestro reflexivo la teoría queda reconstruida por la reflexión sobre la práctica y los problemas prácticos se resuelven con la teoría contextualizada, adquiriendo esta una mayor significatividad.

\subsection{Cuestionario final de autoevaluación y análisis de resultados}

El objetivo general del cuestionario fue conocer cómo los estudiantes percibieron la evolución de su reflexividad a lo largo de las cinco semanas de prácticas mediante la utilización del modelo propuesto de PR. Simultáneamente, se pretendió que valoraran, desde su perspectiva, el modelo utilizado de PR tras su experimentación sistemática en un periodo de cinco semanas consecutivas. La principal preocupación a la hora de elaborar y formular las preguntas consistió en que estas fueran relevantes para el objeto de nuestro estudio y nos reportaran la valoración de los participantes acerca de la prueba piloto que protagonizaron. Principalmente nos interesaba su auto-evaluación acerca de los procesos reflexivos y si consideraban que el modelo les había 
facilitado la conformación de la competencia reflexiva en la práctica profesional. También nos interesaba recabar información abierta sobre qué aspectos consideraban mejorables del modelo para su posible revisión y mejora. El cuestionario se aplicó a las estudiantes una vez que finalizaron sus prácticas. Se les informó que el instrumento pretendía recoger la información referente a su propia valoración y percepción -subjetiva y objetivaacerca de su capacidad reflexiva a lo largo de la experimentación de la metodología y modelo de la PR. También se les informó que el estudio de sus valoraciones personales era parte de la investigación que realiza nuestro Departamento para evaluar el modelo de PR utilizado y su posible mejora en relación con la formación profesional del maestro.

Con el fin de recoger la información básicamente cualitativa, los 32 ítems del cuestionario se agruparon en torno a estos cinco criterios: formación reflexiva, articulación teoría-práctica (durante sus prácticas), modelo metodológico de PR, sistema de evaluación de la PR y observaciones y cuestiones abiertas (no ítems cerrados).

Se apuntó en el cuadro esquemáticamente la información que la docente-investigadora necesitaba recoger con este cuestionario. En el conjunto de la recogida de datos, este instrumento adquirió un valor principal para la investigación evaluativa que se realizó. Se sintetizó el objeto de la información que se pretendía recoger cada bloque de ítems a los que responde el estudiante.

Tabla 2. Bloques temáticos

\begin{tabular}{|c|c|}
\hline $\begin{array}{c}\text { Bloques temáticos del } \\
\text { cuestionario } \\
\text { final de las estudiantes }\end{array}$ & $\begin{array}{l}\text { Objeto de los Ítems a los que } \\
\text { responden las estudiantes }\end{array}$ \\
\hline $\begin{array}{l}\text { A. Formación Reflexiva (6 } \\
\text { ítems) }\end{array}$ & $\begin{array}{l}\text { Valorar si ha incrementado su } \\
\text { reflexividad en torno a la práctica } \\
\text { escolar }\end{array}$ \\
\hline $\begin{array}{l}\text { B. Articulación } \\
\text { Teoría-Práctica } \\
\text { (6 ítems) }\end{array}$ & $\begin{array}{l}\text { Conocer si esta experiencia ha } \\
\text { contribuido a articular mejor } \\
\text { conocimiento teórico y } \\
\text { conocimiento práctico. }\end{array}$ \\
\hline $\begin{array}{l}\text { Modelo de Práctica Reflexiva } \\
\text { (14 ítems) }\end{array}$ & $\begin{array}{l}\text { Valorar los distintos elementos } \\
\text { del modelo. }\end{array}$ \\
\hline $\begin{array}{l}\text { Sistema de evaluación del } \\
\text { Practicum (6 ítems) }\end{array}$ & $\begin{array}{c}\text { Evaluar personalmente el sistema } \\
\text { de trabajo y de evaluación a través } \\
\text { del portafolios o carpeta de } \\
\text { aprendizaje. }\end{array}$ \\
\hline $\begin{array}{l}\text { Observaciones y cuestiones } \\
\text { abiertas ( } \sin \text { ítems). }\end{array}$ & $\begin{array}{l}\text { Aportaciones para la mejora y } \\
\text { revisión del modelo y valoración } \\
\text { general de la experiencia. }\end{array}$ \\
\hline
\end{tabular}

\section{RESULTADOS}

Se presenta a continuación el cuestionario de 32 ítems aplicado, incluyendo a la vez los resultados obtenidos de las respuestas de los estudiantes, expresados en porcentajes.

Tabla 3. Sobre la formación reflexiva

\begin{tabular}{|c|c|c|c|c|}
\hline \multirow{2}{*}{ ÍTEMS } & Mucho & Bastante & Algo & Nada \\
\hline & $\%$ & $\%$ & $\%$ & $\%$ \\
\hline $\begin{array}{l}\text { 1. } \mathrm{He} \text { aprendido } \mathrm{a} \\
\text { reflexionar sobre mi } \\
\text { práctica. }\end{array}$ & 60,00 & 40,00 & 0,00 & 0,00 \\
\hline $\begin{array}{l}\text { 2. He aprendido a partir } \\
\text { de la práctica de mis } \\
\text { compañeras } \\
\text { seminario. }\end{array}$ & 53,33 & 46,67 & 0,00 & 0,00 \\
\hline $\begin{array}{l}\text { 3. He mejorado mi } \\
\text { formación práctica. }\end{array}$ & 40,00 & 46,67 & 13,33 & 0,00 \\
\hline $\begin{array}{l}\text { 4. Estas prácticas han } \\
\text { sido reflexivas para mí. }\end{array}$ & 53,33 & 46,67 & 0,00 & 0,00 \\
\hline $\begin{array}{lr}\text { 5. Esta } & \text { experiencia } \\
\text { incrementa } & \text { mi } \\
\text { profesionalidad docente. }\end{array}$ & 53,33 & 46,67 & 0,00 & 0,00 \\
\hline $\begin{array}{l}\text { 6. Me he planteado la } \\
\text { necesidad de continuar } \\
\text { formándome como } \\
\text { maestro acabada la } \\
\text { carrera y a lo largo de la } \\
\text { vida profesional. }\end{array}$ & 80,00 & 13,33 & 6,67 & 0,00 \\
\hline
\end{tabular}

Tabla 4. Sobre la articulación de teoría y práctica

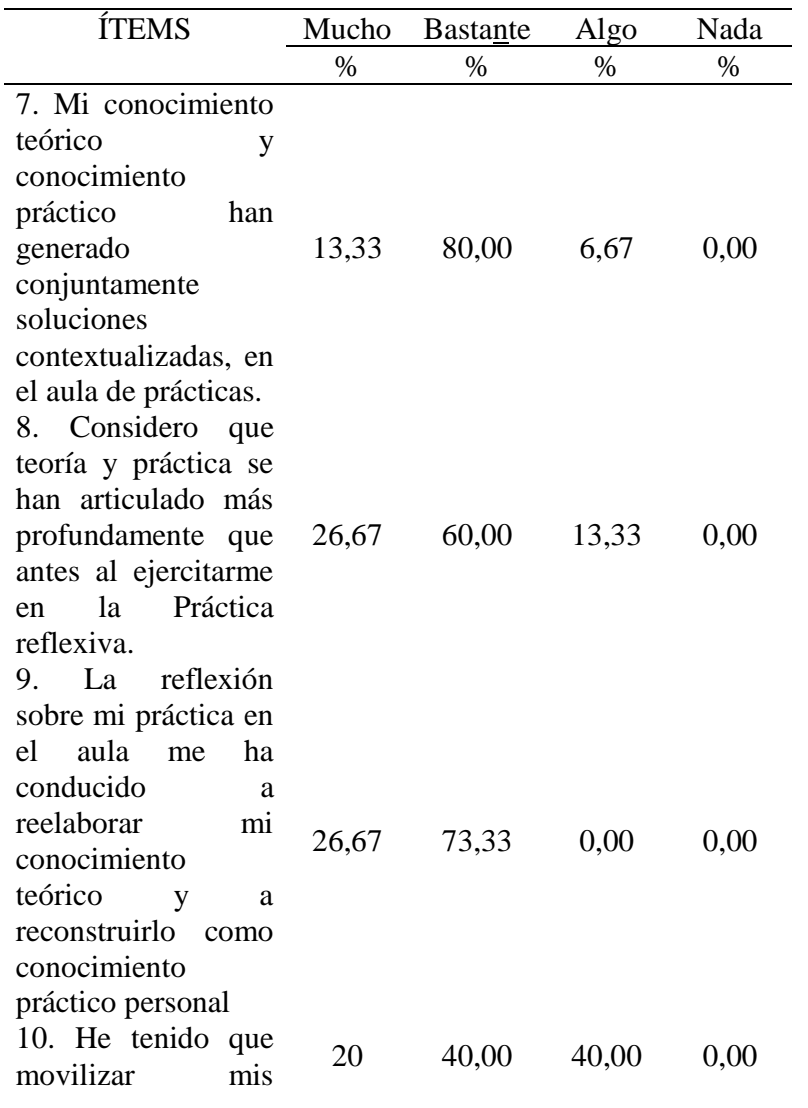


conocimientos

teóricos para

reaccionar y decidir

cómo abordar

cuestiones

pedagógicas en el

aula.

11. La lectura de

documentos teóricos

me ha ayudado a la

reflexión y la

$26,67 \quad 46,67 \quad 26,67 \quad 0,00$

formación práctica.

12. En ocasiones las

situaciones prácticas

me han incitado a

aumentar mi

formación.

Tabla 5. Sobre el método R5 de Práctica Reflexiva

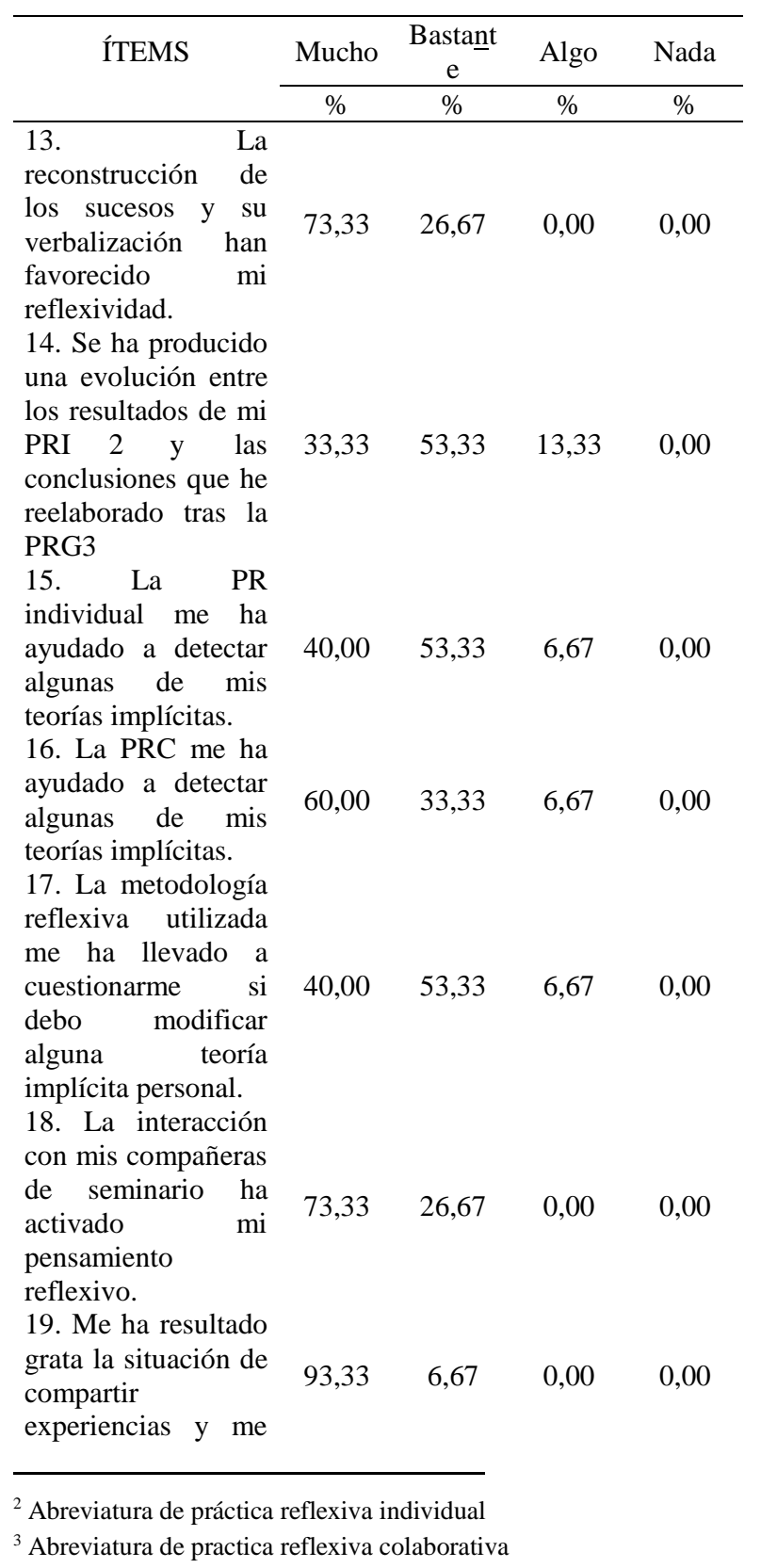

he sentido bien con

ellas en el

seminario.

20. Me ha resultado incómoda la

situación de $\quad 0,00 \quad 0,00 \quad 0,00 \quad 100,0$

compartir

experiencias.

21. La práctica reflexiva individual me ha ayudado la detectar algún elemento emocional mío que ha intervenido en mis actuaciones en el aula.

22. La práctica reflexiva grupal me ha ayudado a detectar algún elemento emocional mío que ha intervenido en mis actuaciones en el aula.

23. Las experiencias particulares compartidas me han permitido llegar a una cierta teorización.

24. $\mathrm{Ha}$ sido satisfactorio el asesoramiento de tutor/a de la universidad durante las prácticas.

25. He tenido el soporte virtual $y$ personal de la universidad:

tutorización,

orientación,

instrumentos,

consultas $\mathrm{y}$

seguimiento online).

26. Los seminarios

de este Practicum

me han parecido

útiles. $\begin{array}{llll}0,00 & 66,67 & 33,33 & 0,00\end{array}$

$46,67 \quad 53,33 \quad 0,00 \quad 0,00$

Tabla 6. Portafolio (estrategia de aprendizaje y de evaluación)

\begin{tabular}{lrcccc}
\hline \multicolumn{1}{c}{ ÍTEMS } & & Mucho & $\begin{array}{c}\text { Bastant } \\
\text { e }\end{array}$ & Algo & Nada \\
\cline { 2 - 6 } & & $\%$ & $\%$ & $\%$ & $\%$ \\
\hline $\begin{array}{l}27 . \quad \text { El sistema de } \\
\text { trabajo }\end{array}$ & & & & \\
portafolio te & ha & 33,33 & 60,00 & 6,67 & 0,00 \\
$\begin{array}{l}\text { facilitado } \\
\text { reflexión. }\end{array}$ & la & & & & \\
$\begin{array}{l}\text { 28. Prefiero el } \\
\text { sistema del trabajo y }\end{array}$ & 46,67 & 53,33 & 0,00 & 0,00
\end{tabular}




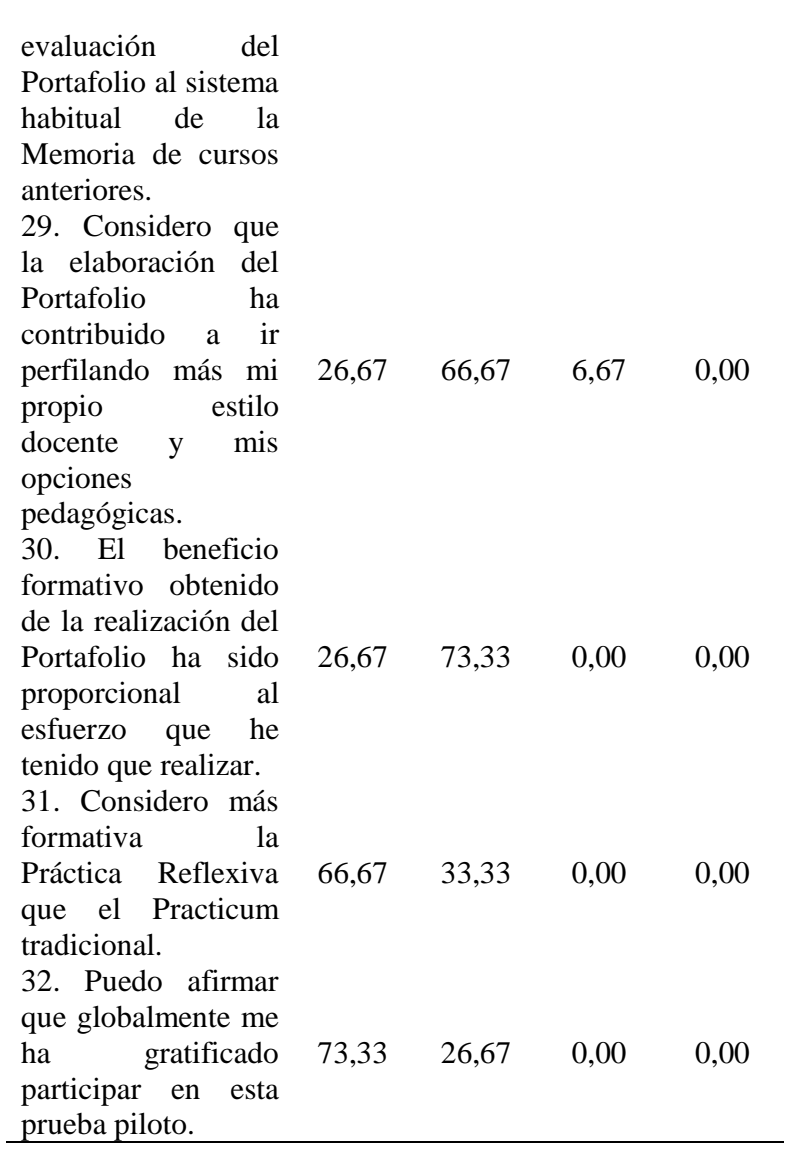

El análisis descriptivo de los resultados obtenidos de los cuestionarios finales señala, de forma relevante, una valoración positiva de la experiencia y del modelo utilizado. Las categorías de respuesta más elevada son las de "mucho" y "bastante" y los resultados son por sí mismos elocuentes y no precisan de mayores comentarios globales respecto al amplio margen que alcanza el sí general en la valoración realizada por las estudiantes.

En línea con el paradigma cualitativo de esta experimentación, es obligado realizar análisis interpretativo de la información obtenida. En esta fase hemos de realizar inferencias e interpretaciones orientadas a la comprensión de los procesos reflexivos que la experimentación del modelo ha propiciado en las estudiantes según su perspectiva y valoración. La valoración interpretativa se presenta sobre los cuatro los bloques temáticos del cuestionario para evitar extendernos excesivamente en los detalles.

\section{DISCUSIÓN}

Los resultados finales del bloque sobre la formación reflexiva de las participantes nos permiten afirmar que, desde la perspectiva de las estudiantes, este Practicum innovador orientado a la PR ha contribuido muy considerablemente al desarrollo de su capacidad reflexiva.

Los resultados nos conducen a comprender que las participantes aprecian personalmente el aprendizaje realizado y el resultado formativo que les ha reportado.

El ítem que destaca particularmente es el $\mathrm{n}^{\circ} 6$, en el que catorce participantes se convencen durante las prácticas de la urgente necesidad de incrementar su formación al finalizar su carrera y a lo largo de todo su futuro profesional. La reflexión sobre su propia práctica les ha permitido detectar que precisan aprender más para resolver profesionalmente las situaciones prácticas en el aula y manifiestan su intención de seguir formándose.

A tenor de estos resultados podemos concluir que, en cuanto a la competencia reflexiva y al desarrollo de la reflexividad de las alumnas participantes en esta PR, su valoración es altamente positiva, a pesar de la novedad e incertidumbre de ser unas prácticas muy distintas a las realizadas en el resto de la carrera.

En el segundo bloque temático se buscó conocer cómo las participantes han vivenciado a nivel subjetivo la articulación de su conocimiento teórico y práctico durante sus prácticas en el contexto del aula. A las participantes les costó responder con seguridad a estos ítems por resultarles cuestiones de difícil valoración al estar relacionados con los procesos del pensamiento docente, realidad que aún les resulta algo abstracta. Los resultados nos llevan a interpretar que, en términos generales, la Práctica Reflexiva ha exigido a las practicantes movilizar simultáneamente conocimiento teórico y conocimiento práctico.

Los resultados muestran que algunas participantes no han advertido una evolución positiva notoria en la articulación de teoría y práctica durante sus prácticas.

Esta experiencia ha mostrado que el conocimiento teórico adquiere una mayor significatividad para el estudiante cuando lo precisa para resolver cuestiones prácticas en el escenario profesional.

En cuanto al bloque sobre el modelo de PR utilizado, los resultados muestran que la mayoría de las participantes valoraron positivamente el modelo utilizado para la PR, así como los elementos metodológicos que lo estructuran. Con los resultados se comprende el valor de la reconstrucción mental de los sucesos vividos en el aula, y de su verbalización posterior para promover la capacidad y formación reflexiva de quien utiliza el modelo. Se encuentra que el modelo propicia nuevos 
aprendizajes prácticos y un mejor autoconocimiento metacognitivo de las actuaciones docentes en el aula.

A partir de los resultados se entiende que la reflexión grupal se valora como el elemento de mayor ayuda para detectar aspectos inconscientes de su actividad escolar, de modo que de PR individual se transforma en PR grupal, con el enriquecimiento que produce la interacción con las demás y el poder, a su vez, participar indirectamente en la PR de otros sin haberla vivido directamente. En este sentido, los resultados notablemente más altos son los referentes a dos puntos de la experiencia:

- Los seminarios vividos como activadores del pensamiento reflexivo y el enriquecimiento a través de la convivencia con los otros.

- El valor de la tutorización durante la PR.

En cuanto al bloque de los portafolios, estos lograron evaluarse como estrategias de formación y aprendizaje reflexivo y como instrumentos de evaluación de la formación reflexiva y práctica en el aula escolar. Se concluyó que el trabajo del portafolio favorece la reflexión e induce a ir modelando y definiendo el estilo del docente. Los estudiantes prefirieron el portafolio reflexivo a la memoria tradicional, considerándolo de mayor utilidad formativa.

\section{CONCLUSIONES}

Analizados los resultados de la implementación del Método R5 de PR en el Practicum 3 de Educación Primaria e Infantil, se presentan las conclusiones valorativas y evaluativas.

Las estudiantes evalúan así el modelo de PR implementado:

- Método muy práctico, no teórico y asequible.

- Ha resultado muy útil.

- El modelo enseña a reflexionar sobre la propia práctica y a aprender de ella.

- Las fases propuestas por Schön han sido de gran utilidad para aprender a reconstruir, analizar y reflexionar las situaciones del aula.

- Los seminarios de reflexión grupal y colaborativa son muy formativos.

- La experiencia ha ayudado a concienciarse de las teorías implícitas y factores emocionales y de su incidencia real en sus actuaciones $y$ reacciones en el aula escolar.

- La interacción y contraste han sido las estrategias de mayor incidencia formativa.
- El modelo ha movilizado en las estudiantes el conocimiento teórico y práctico para su intervención educativa.

- El aprendizaje logrado es más profundo, al ser teórico y práctico a la vez.

- Han aprendido a reflexionar individual y colectivamente.

- Este modelo hace más interesantes y formativas las prácticas.

- El modelo innovador contribuye muy directamente a la preparación profesional.

Por otra parte, cabe añadir que el modelo experimentado ha resultado más formativo porque ha exigido del alumnado una implicación profunda en el aprendizaje experiencial que implicaba su propia reflexión y que se enriquecía con la reflexión de sus compañeras de seminario. Comparado con la orientación tradicional del Practicum se ha logrado unas prácticas escolares más formativas y más exigentes a nivel intelectual por parte de los estudiantes. Estos apenas han tenido que realizar tareas burocráticas en la escuela de prácticas y han centrado su aprendizaje en la inmersión en el aula educativa y en la reflexión sobre sus propias actuaciones. En palabras suyas

ha sido una práctica mucho más agradable y formativa a nivel personal y profesional pero hay que reconocer que nos ha supuesto menos trabajo mecánico pero nos ha exigido un trabajo intelectual y mucho mayor que otros años. Ha implicado más dimensiones de nuestra persona. Pero hay que reconocer que hemos aprendido mucho más y de forma mucho más agradable (Cuestionario final de una de las estudiantes).

Para concluir se presenta la evaluación final -que recoge también la evaluación continuada a lo largo de todo el proceso- del modelo experimentado con relación a los objetivos formativos pretendidos por parte de la docente y tutora:

1. El Método R5 de PR ha enseñado a los estudiantes a reflexionar sobre su práctica docente.

2. La PR metodológica les ha enseñado a ser docentes reflexivos durante sus prácticas.

3. La reconstrucción a posteriori de las situaciones vividas en el aula ha sido uno de los elementos que más ha contribuido a su mejora personal y profesional.

4. La interacción y contraste con sus compañeras durante los seminarios la han valorado muy 
particularmente: les ha abierto panorámicas para mejorar $\mathrm{y}$ ha revalorizado la necesidad de mejorar sus conocimientos teóricos.

5. Lo que los estudiantes más han apreciado del método es la posibilidad de compartir experiencias en los seminarios semanales con sus compañeras; consideran que les ha reportado un incremento de su formación práctica. Esto constituye un buen presagio para sistematizar en las escuelas un trabajo docente más cooperativo.

6. Consideran valiosa la PR como metodología para su formación y competencia profesional.

7. La PR que han experimentado es considerada más formativa y didáctica que los modelos que han experimentado en los anteriores Practicum de la carrera.

8. Su apreciación global de la experiencia es muy positiva y agradable tanto desde el punto de vista formativo como humano.

9. El portafolio como estrategia de trabajo y de evaluación ha contribuido a hacer más reflexivos a las estudiantes que lo han realizado durante esta experiencia.

Las conclusiones permiten inferir que el modelo de PR implementado ha incidido positivamente en la formación reflexiva y práctica de las estudiantes de la muestra y proyecta luz para la toma de decisiones en la orientación de los Practicum del Grado de Educación Infantil y Primaria.

Llegados al momento final la honestidad investigadora obliga a recordar las principales limitaciones que se derivan del objeto de investigación y del método utilizado para investigar. En cuanto al objeto pueden señalarse la complejidad y la subjetividad para llevar a la práctica con estricto rigor alguna de las etapas de la investigación; la evaluación de personas en un contexto real y definido, la validez ecológica -no generalizable- de esta experimentación cuyos resultados están impregnados de elementos contextuales. Y en relación con el método utilizado, recordar los límites propios de la investigación evaluativa: el riesgo de subjetividad, el riesgo de sesgo o parcialidad en las observaciones e informes y la posible influencia del marco referencial de la investigadora que la ha llevado a cabo.

\section{REFERENCIAS}

Domingo, A., y Anijovich, R. (2017). Práctica Reflexiva: Escenarios y horizontes. Avances en el contexto internacional. Buenos Aires: AIQUE.
Domingo, A. (2013). Práctica Reflexiva para docentes. De la reflexión ocasional a la reflexión metodológica. Saarbrücken: Ed. Publicia.

Schön, D. A. (1987). La formación de profesionales reflexivos. Hacia un nuevo diseño de la enseñanza y el aprendizaje de las profesiones. Barcelona: Paidós.

Vygotski, L. S. (1979). El desarrollo de los procesos psicológicos superiores. Barcelona: Crítica. 